\title{
PREVALÊNCIA DE LESÕES DENTÁRIAS EM EXAMES RADIOGRÁFICOS EM UMA POPULAÇÃO AMAZÔNICA
}

\section{PREVALENCE OF DENTAL INJURIES IN RADIOGRAPHIC EXAMINATIONS IN AN AMAZONIAN POPULATION}

Analú Corrêa de Sousa', Jéssica Torres Ferreira', Kunihiro Saito', Wagner Almeida de Andrade', Armando Costa Ferreira', Pedro Luiz de Carvalho'*

'Universidade Federal do Pará, Instituto de Ciências da Saúde, Faculdade de Odontologia, Belém, Pará, Brasil

*Autor correspondente: Rua Augusto Corrêa, 01, Guamá, Belém -PA, Brasil, CEP: 66075-110, Fone: +5591993933358, e-mail: pedrolc@ufpa.br

\section{RESUMO}

As alterações dentárias são ocasionadas por processos iatrogênicos, traumáticos e cariogênicos, muitas vezes são diagnosticadas através de seus sinais e sintomas clínicos, sem necessidade de radiografias. Os exames radiográficos permitem ao profissional avaliar áreas que não podem ser examinadas clinicamente, tal como, raízes e cavidades pulpares dos dentes. O presente trabalho tem como propósito verificar a prevalência de lesões dentárias em exames radiográficos periapicais digitais de pacientes em uma Clínica Radiológica, correlacionando as variáveis de idade, gênero e o dente envolvido. A amostra da pesquisa constituiu-se de exames radiográficos periapicais com imagem de lesões dentárias sugestiva de: cárie, desgaste dentário (abrasão, atrição e erosão), fratura coronária, nódulo pulpar, mineralização pulpar, reabsorção radicular interna, reabsorção radicular externa, hipercementose, fratura radicular, perfuração radicular e raiz residual. Os dados obtidos foram tabulados utilizando os programas Microsoft Office Excel e Bioestat 5.0 ao nível de significância de 5\%. Dos exames avaliados 719 radiografias apresentaram imagens de lesões dentárias, uma ou mais, correspondente a 302 pacientes, 186 (61,6\%) do gênero feminino e $116(38,4 \%)$ masculino. O número de lesões presentes apresentou-se com mais frequência em dentes com imagem sugestiva de cárie $(46,1 \%)$, a seguir desgaste dentário $(24,9 \%)$ e raiz residual (11,9\%). A faixa etária mais afetada foi a de 51 a 60 anos com um total de 251 lesões, sendo mais incidente o desgaste dentário no sexo masculino. Assim, foi possível concluir que: o gênero feminino foi o mais acometido $(61,6 \%)$, a faixa etária mais incidente foi de 51 a 60 anos; a imagem radiográfica de cárie foi a alteração de alta prevalência, os dentes mais acometidos foram os incisivos centrais superiores.

Palavras-Chave: Diagnóstico por imagem. Radiografia dentária. Radiografia panorâmica. Traumatismo dentário.

\section{ABSTRACT}

The dental abnormalities are caused by iatrogenic processes, traumatic and cariogenic, often are diagnosed by their clinical signs and symptoms, without the necessity of $x$-rays. The radiographic examinations allow the professional to assess areas that may not be examined clinically, as roots, pulpares cavities of the teeth. The present study aims to investigate the prevalence of dental injuries in periapical radiographic examinations of patients in a digital Radiology Clinic, 
correlating the variables of age, gender, and the tooth involved. The research sample consisted of periapical radiographic examination with image of dental lesions suggestive of: dental caries, dental wear (abrasion, atrição and erosion), coronary fracture nodule mineralization, pulp, pulp, root resorption internal, external root resorption, hipercementose, root, root fracture drilling and residual root. The data obtained were tabulated using the programs Microsoft Office Excel and Bioestat 5.0 at a significance level of $5 \%$. The examinations evaluated 719 chest $x$-rays showed images of dental injuries, one or more corresponding to 302 patients, 186 (61.6\%) of the female gender and $116(38.4 \%)$ male. The number of lesions present, presenting with more frequency of teeth with an image suggestive of caries $(46.1 \%)$, the following dental wear $(24.9 \%)$ and residual root (11.9\%). The age group most affected was 51 to 60 years with a total of 251 lesions, being more incident the dental wear in males. Thus, it was possible to conclude that: the female gender was the most affected (61.6\%), the age group most incident was from 51 to 60 years; the radiographic image of caries was the change of high prevalence, the most affected teeth were the maxillary central incisors.

Keywords: Diagnostic imaging, radiography dental, radiography panoramic, tooth injuries.

\section{INTRODUÇÃO}

As estruturas dentárias podem ser perdidas por lesões que correspondem as modificações dos tecidos dentário, essas alterações podem decorrer em caso de uma injúria na coroa ou raiz do dente, na maioria das vezes são determinadas por processos iatrogênicos, traumáticos, ou ainda, cariogênicos. Exames por imagens, como radiografias periapicais, podem revelar uma variedade de achados como áreas de destruição ou desgaste, e por vezes, associada a lesões ósseas periapicais.

O exame radiográfico representa uma alternativa para avaliar as lesões dentárias, sendo indicado como rotina na consulta clínica odontológica. Este exame tem a capacidade de captar toda a área óssea e dentada que compõe o sistema estomatognático do indivíduo ${ }^{1}$. A rigorosa observação do exame radiográfico assume um papel valioso na determinação do diagnóstico, do prognóstico e na avaliação do resultado do tratamento.

A solicitação de exames radiográficos como rotina no tratamento dos pacientes vem conquistando cada vez mais importância na odontologia, e dessa forma, com um maior número de realizações de documentações radiográficas, os achados de lesões dentárias são mais comumente encontrados. Apesar disso, algumas pesquisas foram realizadas com o objetivo de determinar a prevalência das lesões dentárias nas arcadas dentárias ${ }^{2-10}$. Tais lesões assumem caráter de relevância, uma vez que, apresentam considerável prevalência na população e devido ao seu desenvolvimento assintomático na maior parte dos casos, os achados referentes a essas lesões são basicamente radiográficos ${ }^{11}$. A análise adequada da radiografia vem sinalizar uma condição clínica importante de qualquer problema que possam ocorrer nessas estruturas.

Uma avaliação radiográfica adequada poderá auxiliar na conduta terapêutica a ser utilizada nos casos de lesões dentárias, também facilitar na prevenção das consequências nos danos estéticos para o paciente, dentre outras causas, além de permitir avaliar a extensão e a relação anatômica dessas lesões com as estruturas dentárias. 
O propósito deste estudo foi verificar a prevalência de lesões dentárias em exames radiográficos periapicais digitais de pacientes em uma Clínica Radiológica de Belém-PA, além de correlacionar as variáveis idade e gênero dos pacientes da pesquisa, e o dente envolvido nas imagens dos exames radiográficos periapicais digitais com diagnóstico radiográfico compatível com alterações dentárias.

\section{MATERIAL E MÉTODOS}

Esta pesquisa foi aprovada pelo Comitê de Ética em Pesquisa do Instituto de Ciências da Saúde da Universidade Federal do Pará sob o número 1.423.820.

Realizou-se um estudo radiográfico retrospectivo de 2.000 exames periapicais digitais de pacientes atendidos em Clínica Radiológica Privada, localizada na cidade de Belém - PA, realizados no período de janeiro a junho de 2016.

A amostra constituiu-se de exames radiográficos periapicais com imagem de lesões dentárias sugestiva de: cárie, desgaste dentário (abrasão, atrição e erosão), fratura coronária, nódulo pulpar, mineralização pulpar, reabsorção radicular interna, reabsorção radicular externa, hipercementose, fratura radicular, perfuração radicular e raiz residual (Tabela 1).

Foram considerados os seguintes critérios de inclusão na amostra: exames com informações sobre gênero e idade dos pacientes e radiografias com aceitáveis padrões de qualidade da imagem radiográfica.

Os exames radiográficos foram analisados em monitor de computador em ambiente com pouca luminosidade. Foram elaboradas fichas especialmente para a anotação dos dados obtidos que continham nome do paciente, gênero, idade e presença de imagem sugestiva de lesões dentárias, bem como os dentes relacionados; efetuadas por dois examinadores. Quando houve divergência, os examinadores discutiram e determinaram a alteração dentária.

Nos exames com imagens compatíveis com lesões dentárias, quando necessário, foi realizado tratamento digital adequando-se contraste, brilho, realce dos contornos e representação das imagens radiográficas em relevo. O editor de imagens utilizado foi o Adobe Photoshop CS3.

Tabela 1 - Aspectos radiográficos das lesões consideradas no estudo.

\begin{tabular}{|c|c|c|}
\hline \multicolumn{2}{|l|}{ Lesão Dentária } & Descrição radiográfica: \\
\hline \multicolumn{2}{|l|}{ Cárie } & $\begin{array}{l}\text { Imagem radiolúcida na coroa ou raiz do dente assumindo forma irregular e } \\
\text { margens difusas. }\end{array}$ \\
\hline \multirow{3}{*}{ Desgaste dentário } & Abrasão & $\begin{array}{l}\text { Imagem radiolúcida semilunar na região cervical dos dentes, com cavidades } \\
\text { pulpares obliteradas parcial ou totalmente. }\end{array}$ \\
\hline & Atrição & $\begin{array}{l}\text { Imagem de uma coroa mais curta, superfície normalmente plana, com } \\
\text { redução da câmara pulpar e espessamento do ligamento periodontal. }\end{array}$ \\
\hline & Erosão & $\begin{array}{l}\text { Área radiolúcida de forma côncava (cunha) na coroa, com margens bem } \\
\text { definidas. }\end{array}$ \\
\hline \multicolumn{2}{|l|}{ Fratura coronária } & Área radiolúcida com aspecto de descontinuidade do contorno coronário. \\
\hline \multicolumn{2}{|l|}{ Nódulo pulpar } & Massa radiopaca arredondada ou ovalada na cavidade pulpar. \\
\hline \multicolumn{2}{|c|}{ Mineralização pulpar } & Obliteração parcial ou total da cavidade pulpar. \\
\hline \multicolumn{2}{|c|}{ Reabsorção radicular interna } & $\begin{array}{l}\text { Área radiolúcida arredondada, oval ou alongada na raiz ou coroa, sendo } \\
\text { contínuas com a imagem da câmara ou canal pulpar. }\end{array}$ \\
\hline \multicolumn{2}{|c|}{ Reabsorção radicular externa } & $\begin{array}{l}\text { Diminuição do tamanho da raiz, mantendo o espaço pericementário e } \\
\text { lâmina dura intactos, envolvendo o ápice e, ocasionalmente, a porção } \\
\text { lateral radicular. }\end{array}$ \\
\hline
\end{tabular}

continua 
conclusão

\begin{tabular}{l|l} 
Lesão Dentária & Descrição radiográfica: \\
Hipercementose & $\begin{array}{l}\text { Aumento da radiopacidade do cemento, com consequente aumento de } \\
\text { volume radicular, lâmina dura e espaço do ligamento periodontal preservados. }\end{array}$ \\
\hline Fratura radicular & Linha radiolúcida bem definida confinada aos limites anatômicos da raiz. \\
\hline Perfuração radicular & $\begin{array}{l}\text { Imagem radiolúcida na porção radicular, com perda de detalhe da lâmina } \\
\text { dura acompanhada de uma área de rarefação óssea circunscrita. }\end{array}$ \\
\hline Raiz residual & $\begin{array}{l}\text { Imagem radiopaca de limites regulares, geralmente com forma cônica, longo } \\
\text { eixo na vertical, podendo apresentar imagem radiolúcida do conduto e } \\
\text { espaço periodontal. }\end{array}$ \\
\hline
\end{tabular}

Os dados obtidos foram tabulados utilizando o programa Microsoft Office Excel (versão 2019, Microsoft Office Corporation) para frequência e porcentagem das lesões dentárias. A relação entre os grupos etários foi analisada usando o teste qui-quadrado no programa Bioestat 5.0 (Belém, Pará, Brasil) os resultados foram considerados significantes se o $p$-valor foi menor que $5 \%(p<0,05)$.

\section{RESULTADOS}

Foram analisadas 2.000 radiografias periapicais digitais de 370 pacientes, 222 do gênero feminino e 148 masculino com idade acima de 11 anos e com limite máximo de 70 anos. Dos exames avaliados apenas 719 radiografias apresentaram imagens de lesões dentárias, uma ou mais, correspondente a 302 pacientes, sendo que 186 (61,6\%) do gênero feminino e $116(38,4 \%)$ do gênero masculino. Os pacientes foram divididos em seis subgrupos etários: 11 a 20, 21 a 30, 31 a 40, 41 a 50, 51 a 60 e 61 a 70 anos (Tabela 2).

Tabela 2 - Distribuição dos pacientes com exames radiográficos com imagens de lesões dentárias, segundo o grupo etário.

\begin{tabular}{l|l|l|l} 
Grupos etários & $\begin{array}{l}\text { Feminino } \\
\mathbf{N}(\%)\end{array}$ & $\begin{array}{l}\text { Masculino } \\
\mathbf{N}(\%)\end{array}$ & $\begin{array}{l}\text { TOTAL } \\
\mathbf{N}(\%)\end{array}$ \\
\hline 11 a 20 & $21(11,3)$ & $9(7,8)$ & $30(9,9)$ \\
\hline 21 a 30 & $39(21,0)$ & $27(23,3)$ & $66(21,9)$ \\
\hline 31 a 40 & $22(11,8)$ & $24(20,7)$ & $46(15,2)$ \\
\hline 41 a 50 & $47(25,3)$ & $19(16,4)$ & $66(21,9)$ \\
\hline 51 a 60 & $36(19,4)$ & $26(22,4)$ & $62(20,5)$ \\
\hline 61 a 70 & $21(19,4)$ & $11(9,5)$ & $32(10,5)$ \\
\hline TOTAL & $186(100)$ & $116(100)$ & $302(100)$ \\
\hline$X^{2}=7,88, p=0,1628$. & & &
\end{tabular}


Figura 1 - Distribuição das imagens de lesões dentárias

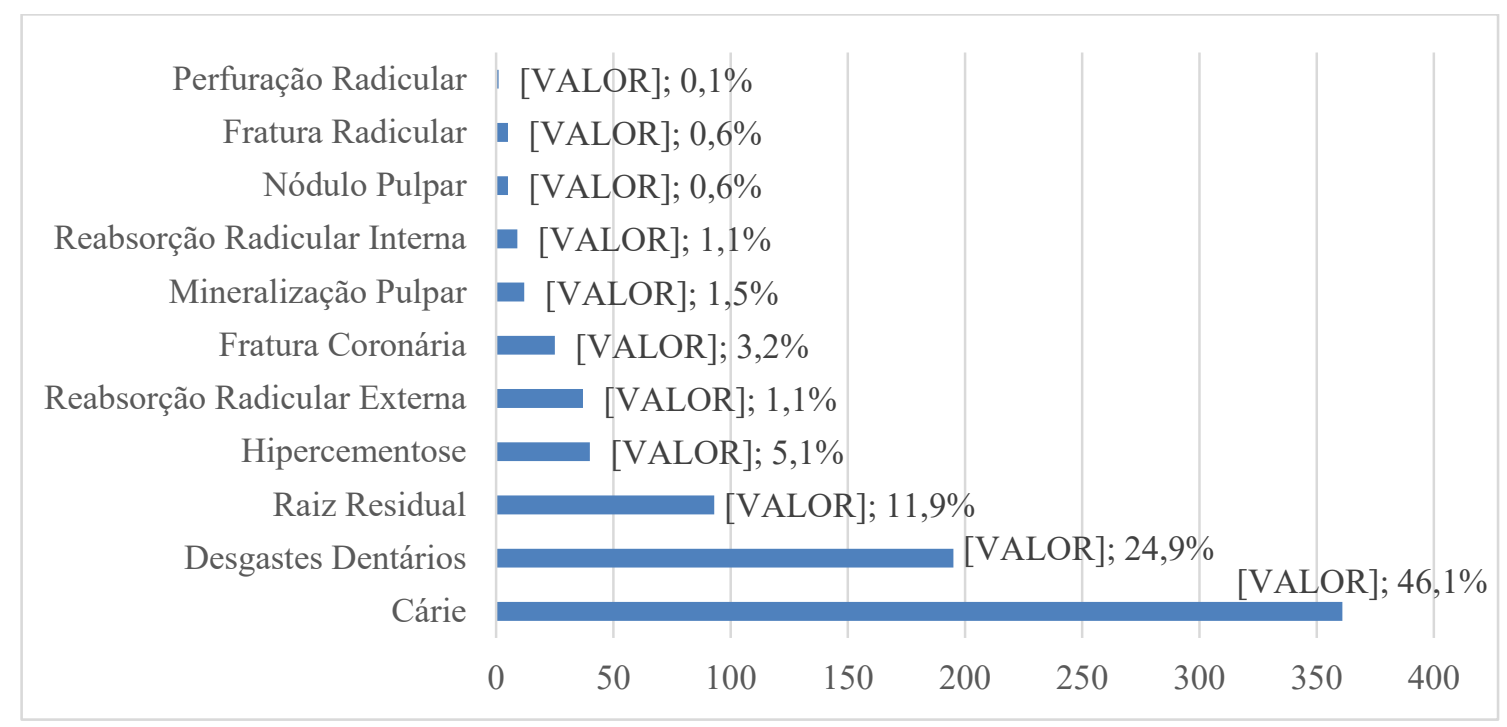

Ao analisar a figura 1, observamos o número das lesões presentes, constatou-se com mais frequência nos dentes com imagem sugestiva de cárie (46,1\%), a seguir desgaste dentário (24,9\%), raiz residual (11,9\%), hipercementose (5,1\%), reabsorção radicular externa $(4,7 \%)$, fratura coronária $(3,2 \%)$, e outras lesões como: nódulo pulpar, mineralização pulpar, reabsorção radicular interna, fratura radicular e perfuração radicular tiveram incidência abaixo de 1,5\%.

Tabela 3 - Distribuição das imagens de lesões dentárias, segundo o grupo etário e gênero

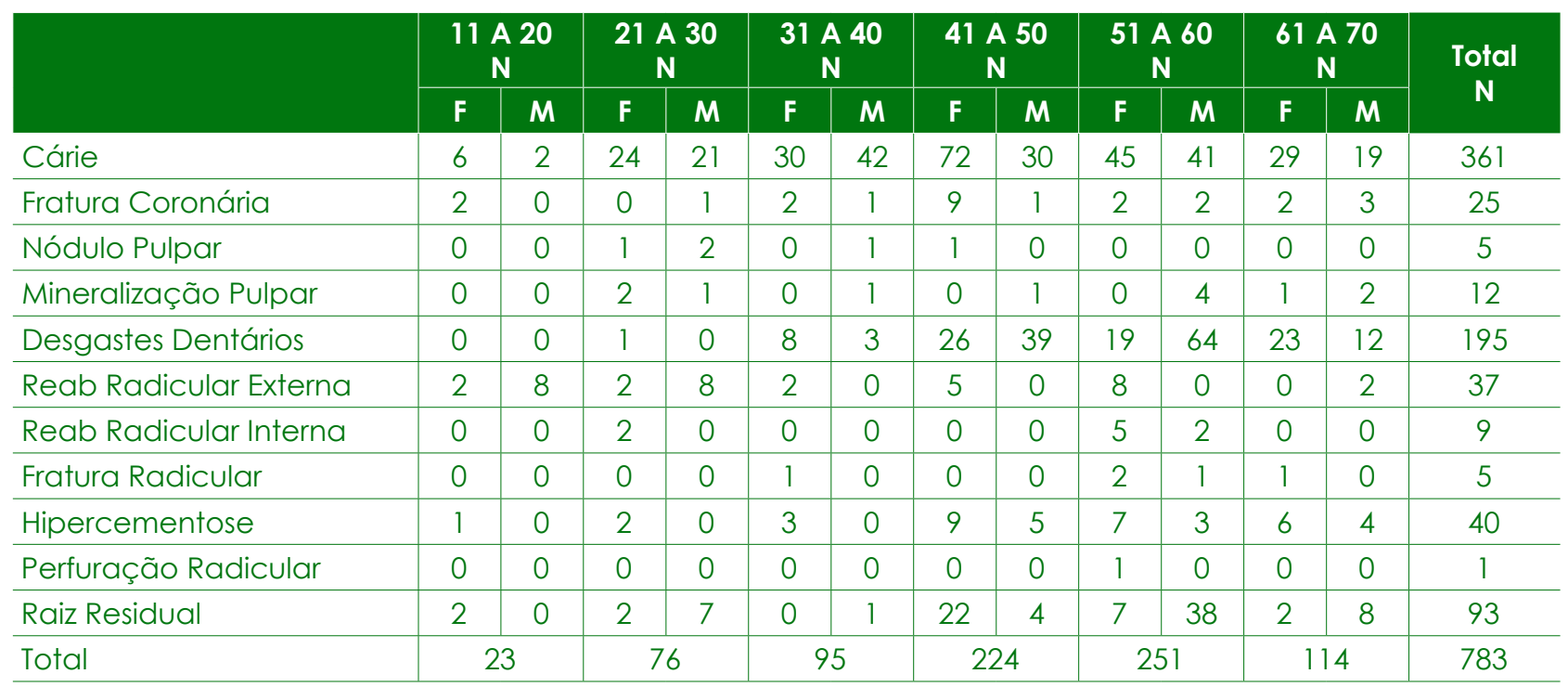

Na tabela 3 observar-se que a faixa etária mais afetada foi a de 51 a 60 anos com um total de 251 lesões, sendo mais incidente o desgaste dentário no sexo masculino. Por outro lado, a faixa etária de 11 a 20 anos foi a menos atingida, apenas 23 lesões, com reabsorção radicular externa no sexo masculino. Já a tabela 4 verifica-se que houve maior incidência de cárie nos incisivos centrais (74), seguido dos primeiros molares (60). 
Tabela 4 - Distribuição das imagens de lesões dentárias, segundo o grupo dentário

\begin{tabular}{l|l|l|l|l|l|l|l|l|l|l|l} 
& $\mathbf{3 M}$ & $\mathbf{2 M}$ & $\mathbf{1}$ & $\mathbf{2 P M}$ & $\mathbf{1 P M}$ & $\mathbf{C}$ & IL & \multicolumn{1}{l}{ IC } \\
\hline Cárie & 17 & 49 & 60 & 41 & 34 & 29 & 57 & 74 & 361 \\
\hline Fratura Coronária & 0 & 4 & 3 & 2 & 1 & 5 & 2 & 8 & 25 \\
\hline Nódulo Pulpar & 1 & 1 & 0 & 1 & 1 & 0 & 1 & 0 & 5 \\
\hline Mineralização Pulpar & 1 & 2 & 6 & 2 & 0 & 0 & 0 & 1 & 12 \\
\hline Desgastes Dentários & 4 & 17 & 10 & 19 & 20 & 45 & 41 & 39 & 195 \\
\hline Reab. Radicular Externa & 0 & 1 & 1 & 1 & 2 & 2 & 12 & 18 & 37 \\
\hline Reab. Radicular Interna & 0 & 0 & 0 & 0 & 0 & 2 & 1 & 6 & 9 \\
\hline Fratura Radicular & 0 & 0 & 2 & 2 & 1 & 0 & 0 & 0 & 5 \\
\hline Hipercementose & 4 & 6 & 6 & 13 & 5 & 1 & 4 & 1 & 40 \\
\hline Perfuração Radicular & 0 & 0 & 1 & 0 & 0 & 0 & 0 & 0 & 1 \\
\hline Raiz Residual & 2 & 7 & 10 & 28 & 20 & 8 & 7 & 11 & 93 \\
\hline TOTAL & 29 & 87 & 99 & 109 & 84 & 92 & 125 & 158 & 783 \\
\hline
\end{tabular}

\section{DISCUSSÃO}

O objetivo deste estudo foi verificar a prevalência de lesões dentárias em exames radiográficos periapicais digitais do acervo de uma Clínica Radiológica de Belém-PA, além de correlacionar as variáveis idade e gênero dos pacientes da pesquisa, o dente envolvido, nas imagens dos exames radiográficos com diagnóstico radiográfico compatível com alterações dentárias.

A amostra foi restrita a exames radiográficos periapicais digitais com imagem sugestiva de: cárie, desgaste dentário (abrasão, atrição e erosão), fratura coronária, nódulo pulpar, mineralização pulpar, reabsorção radicular interna, reabsorção radicular externa, hipercementose, fratura radicular, perfuração radicular e raiz residual.

Um dos pontos observados e que merecem destaque foi a presença considerável do público feminino nos serviços de saúde $(61,6 \%)$ quando comparado ao gênero masculino $(38,4 \%)$ e que tal fato poderia ser explicado pela disponibilidade da mulher em procurar o serviço de saúde. Estes dados são semelhantes a outras análises realizadas no âmbito dos serviços ofertados pelo Sistema Único de Saúde-SUS, evidenciando que a presença do público com essas características não tende a influenciar de forma significativa na satisfação, a depender de como se é destinado o atendimento e resolutividade de seus problemas ${ }^{12}$.

Segundo os resultados deste estudo a presença da imagem de cárie denotou-se o fator mais prevalente nas imagens analisadas perfazendo um total de 46,1\%, valor menor aos índices encontrados por Nóbrega et al. ${ }^{13}(50,2 \%)$ e Frazão et al. ${ }^{14}$ (62,9\%) com estudos clínicos. Esses índices devem-se a complexidade dessa doença, sendo a frequência de escovação e fatores extra biológicos, apontados como fatores que levam ao aumento da prevalência de patologias bucais ${ }^{9}$. Observa-se que os dentes com imagem sugestiva de cárie tiveram um resultado expressivo dentre as lesões dentárias observadas, apesar de uma efetiva odontologia preventiva no estado do Pará, o dente mais incidente foi o incisivo central superior. Porém, Marques et al. ${ }^{8}$ observa que tanto entre os adultos como entre os idosos os dentes posteriores (pré-molares e molares) foram proporcionalmente mais afetados. Não obstante, em uma revisão mais recente Lopez et al. ${ }^{15}$ verificaram-se que os índices de cárie dentária na população idosa vêm caindo nas últimas décadas, e constatou-se uma prevalência em torno de $20 \%$ a $30 \%$, a depender de alguns fatores, como o nível socioeconômico. Idosos com nível socioeconômico 
mais elevado apresentaram uma prevalência maior de cárie, provavelmente devido ao maior número de dentes presentes, quando comparado com idosos em menor nível socioeconômico. O presente estudo avaliou a associação do gênero com a presença de lesão, a cárie foi mais prevalente no sexo feminino (51,6\%) do que o sexo masculino (40,4\%).

O estudo apresentou o valor de 24,9\% para a imagens sugestivas de desgaste dentário, em relação à faixa etária mais atingida independente de lesão foi a de 51 a 60 anos, prevalecendo nos pacientes do sexo masculino. De acordo com Ferreira et al. ${ }^{5}$ características como: a localização geográfica, critérios de diagnóstico, índices, dentes, faixas etárias variáveis, fatores culturais e sociodemográficos, dificultam a comparação entre os estudos e, muitas vezes, impedem o estabelecimento do perfil do problema, tanto no nível mundial quanto ao nacional. Os adultos apresentaram maior incidência dessa alteração, que em geral motiva a busca pelo atendimento odontológico, o que demonstra intensificar o trabalho de prevenção desenvolvido nessa região brasileira. Já as imagens sugestivas de raízes residuais foram observadas em 11,9\% da amostra, mostrando uma frequência inferior à observada por Dias et al..$^{10}(23,6 \%)$ que avaliou 1006 imagens panorâmicas. Já no estudo de Limeira et al. ${ }^{16}$ as raízes residuais foram observadas em $26,7 \%$ da amostra investigada em uma população com características similares a avaliada na pesquisa de Dias et al. ${ }^{10}$.

Durante a análise dos dados obtidos por meio de análise radiográfica foi possível verificar a prevalência de 5,1\% de casos de hipercementose, alteração caracterizada por uma formação excessiva de cemento além do limite fisiológico dos dentes, promovendo um espaçamento anormal do ápice com aspecto arredondado. Essa alteração não corrobora com os registros populacionais de outros locais (0,62\%) em São Paulo ${ }^{17}$ e 3,2\% em Maringá - PR .

O presente estudo buscou avaliar a incidência de imagens sugestivas de fraturas coronárias encontradas em exames de imagem periapicais de pacientes atendidos em uma clínica radiológica privada, dentro do qual os pacientes habitualmente vêm encaminhados de diversos profissionais de consultórios privados. Observou-se uma frequência de 3,2\%, já Carvalho et al. ${ }^{7}$ obtiveram uma frequência de $46,5 \%$.

A reabsorção radicular externa no estudo apresentou incidência de 4,7\%, índice superior ao encontrado por Maues et al. ${ }^{18}$ o valor de 2,9\%. Segundo Sameshima e Asgarifar ${ }^{19}$, a radiografia periapical tem sido o exame mais frequentemente usado para avaliar a reabsorção radicular externa resultante do tratamento ortodôntico devido a sua maior precisão em comparação à radiografia panorâmica.

A imagem sugestiva de mineralização pulpar teve frequência de 1,5\%, porém no estudo de Kumar et al..$^{20}$ apontam que a alta prevalência de calcificações na câmara pulpar em dentes cariados, restaurados e restaurados e cariados também fornece evidências de que a ocorrência de calcificações na câmara pulpar podem ser uma resposta a fatores irritantes de longa duração.

Para Lyroudia et al. ${ }^{21}$ a reabsorção radicular interna é uma entidade relativamente rara, por isso existem poucos trabalhos na literatura que avaliam a sua incidência, fato que foi observado nos nossos achados com incidência de 1,1\%, valor baixo também foi encontrado por Araujo e Lins ${ }^{3}$ em trabalho de análise de prontuários, o que correspondeu a 2,16\% da amostra estudada. Acredita-se que muitos casos a reabsorção interna são detectados em exames radiográficos de rotina.

A frequência de ocorrência de imagens de fraturas radiculares e nódulos pulpares foram 0,6\%. O processo de avaliação de fraturas radiculares torna-se um desafio para o cirurgião dentista. Em vista disso, faz-se necessário o apoio de uma técnica radiográfica adequada, já que, muitas vezes, 
devido à inclinação da fratura ou ao deslocamento dos fragmentos, a sua localização é identificada depois de repetidas incidências radiográficas.

A presença de nódulo pulpar foi de $0,6 \%$ frequência muito baixa ao compararmos com Ranjitkar et $a .^{2}$ que observaram a ocorrência de nódulos pulpares em 10,1\% dos dentes examinados, e Sener et $\mathrm{al}^{4}(4,8 \%)$.

O índice de ocorrência de imagens sugestivas a perfurações radiculares observados foi 0,1\%, valor inferior ao encontrado por Sousa e Rezende ${ }^{22}$ correspondendo a 0,69\%.

\section{CONCLUSÃO}

De acordo com a metodologia empregada e os resultados obtidos foi possível concluir que: o gênero feminino foi o mais acometido (61,6\%), a faixa etária mais incidente foi de 51 a 60 anos; a imagem radiográfica de cárie foi a alteração de alta prevalência, os dentes mais acometidos foram os incisivos centrais superiores.

\section{REFERÊNCIAS}

1. Schwengber MMB, Hennigen TW, Ponzoni D, Puricelli E. Estudo radiográfico da prevalência de lesões periapicais nas arcadas dentárias. Revista da ABO. 2008;16(4):214-7.

2. Ranjitkar S, Taylor JA, Townsend GC. A radiographic assessment of the prevalence of pulp stones in Australians. Aust Dent J. 2002;47(1):36-40.

3. Araújo LCG, Lins CCSA. Prevalência de reabsorção em prontuários de pacientes na clínica de Especialização em Endodontia na UFPE. IDJ, Int J Dent. 2007;6(3):71 -4.

4. Sener S, Cobankara FK, Akgünlü F. Calcifications of the pulp chamber: prevalence and implicated factors. Clin Oral Investig. 2009;13(2):209-15.

5. Ferreira FV, Piovesan C, Praetzel JR, Ardenghi TM. Aspectos clínicos e epidemiológicos da erosão dental na dentição permanente: revisão de literatura. Braz J Health. 2010;1:1-9.

6. Menini AAS, Silva MC, Iwaki LCV, Takeshita WM. Estudo radiográfico da prevalência de anomalias dentárias por meio de radiografias panorâmicas em diferentes faixas etárias. Rev Odontol Univ Cid São Paulo. 2012;24(3):170-7.

7. Carvalho RG, Soares SR, Silva EJNL, Accorsi-Mendonça T, Fonseca OHS, Antunes HS et al. Estudo epidemiológico das fraturas coronárias em pacientes atendidos em um projeto de trauma dental em um período de 6 anos. Rev Bras Odontol. 2013;70(1):4-7.

8. Marques RAA, Antunes JLF, Sousa MLR, Peres MA, Frazão P. Prevalência e extensão da cárie dentária radicular em adultos e idosos brasileiros. Rev Saúde Pública. 2013;47(Supl 3):59-68.

9. Costa FCM, Fernandes LHF, Moura EFF, Aguiar, YPC, Santos FG, Cavalcanti AL. Hábitos de saúde bucal, prevalência de cárie dentária e erosão dentária em adolescentes. Rev Gauch Odontol. 2017;65(3):202-7.

10. Dias ACMS; Medeiros AMC; Freitas YNL; Lima KC; Maia PRL; Oliveira PT. Achados radiográficos em radiografias panorâmicas de idosos: estudo transversal em 1006 pacientes. Rev Port Estomatol Med Dent Cir Maxilofac. 2019;60(2):59-65. 
11. Fenyo-Pereira M. Radiologia odontológica e imaginologia. 2ª ed. São Paulo: Santos, 2019.

12. Rosso JA; Silva RM. Avaliação da qualidade do atendimento em Unidades Primárias de Saúde: comparação de estruturas com e sem a presença de Acadêmicos de Medicina. Ara Catarin Med. 2006:35(2):47-55.

13. Nóbrega A, Moura LFAD, Andrade NS, Lima CCB, Dourado DG, Lima MDM. Impacto da cárie dentária na qualidade de vida de pré-escolares mensurado pelo questionário PedsQL. Ciência \& Saúde Coletiva. 2019;24(11):4031-41.

14. Frazão P, Santos CRI, Benicio DEDA, Marques RAA, Benício MHDA, Cardoso MA, Narvai PC. Cárie dentária em escolares de 12 anos de idade em município sem água fluoretada na Amazônia Ocidental brasileira, 2010. Epidemiol Serv Saúde. 2016;25(1):149-58.

15. Lopez R, Smith PC, Gostemeyer G, Schwendicke F. Ageing, dental caries and periodontal diseases. J Clin Periodontol. 2017;44(Suppl 18):S145-52.

16. Limeira FIR, Melo DP, Gomes DQC, Cavalcanti SALB, Bento PM. Radiographic evaluation of the dental condition of elderly people treated at a Brazilian public university. Braz J Oral Sci. 2013;12(4):352-6.

17. Varoli FP, Warmling LV, Santos KCP, Oliveira JX. Occurence of lesions, abnormalities and dentomaxillofacial changes observed in 1937 digital panoramic radiography. J Health Sci Inst. 2013;31 (3):258-61.

18. Mavés CPR, Nascimento RR, Vilella OV. Reabsorção radicular grave resultante do tratamento ortodôntico: Prevalência e fatores de risco. Dental Press J Orthod. 2015;20(1):52-8.

19. Sameshima GT, Asgarifar KO. Assessment of root resorption and root shape: periapical vs panoramic films. Angle Orthod. 2001;71(3):185-9.

20. Kumar S, Chandra S; Jaiswal JN. Pulp calcifications in primary teeth. J Endod. 1990;16(5):218-20.

21. Lyroudia KM, Dourou VI, Pantelidou OC, Labrianidis T, Pitas IK. Internal root resorption studied by radiography, stereomicroscope, scanning electron microscope, and computerized 3D reconstructive method. Dent Traumatol. 2002;18(3):148-52.

22. Sousa PCF, Rezende TMB. Ocorrência de perfurações radiculares nas clínicas odontológicas integradas da universidade católica de Brasília. Oral Sci. 2012;4(1):18-23. 